\title{
Osteogenic actions of the anti-diabetic drug metformin on osteoblasts in culture
}

\author{
Ana M. Cortizo *, Claudia Sedlinsky, Antonio D. McCarthy, Alcira Blanco, León Schurman \\ Cátedra de Bioquímica Patológica (A.M.C., A.D.M.), Facultad de Ciencias Exactas, Universidad Nacional de La Plata, 1900 La Plata, \\ Argentina and Centro de Endocrinología y Metabolismo (C.S., A.B., L.S.), Hospital Francés, Buenos Aires, Argentina
}

Received 16 December 2005; received in revised form 10 February 2006; accepted 17 February 2006

Available online 28 February 2006

\begin{abstract}
An association has been previously established between uncompensated diabetes mellitus and the loss of bone mineral density and/or quality. In this study, we evaluated the effects of metformin on the growth and differentiation of osteoblasts in culture. Treatment of two osteoblast-like cells (UMR106 and MC3T3E1) with metformin $(25-500 \mu \mathrm{M})$ for $24 \mathrm{~h}$ led to a dose-dependent increase of cell proliferation. Metformin also promoted osteoblastic differentiation: it increased type-I collagen production in both cell lines and stimulated alkaline phosphatase activity in MC3T3E1 osteoblasts. In addition, metformin markedly increased the formation of nodules of mineralization in 3-week MC3T3E1 cultures. Metformin induced activation and redistribution of phosphorylated extracellular signal-regulated kinase (P-ERK) in a transient manner, and dosedependently stimulated the expression of endothelial and inducible nitric oxide synthases (e/iNOS). These results show for the first time a direct osteogenic effect of metformin on osteoblasts in culture, which could be mediated by activation/redistribution of ERK-1/2 and induction of e/ iNOS.
\end{abstract}

(C) 2006 Elsevier B.V. All rights reserved.

Keywords: Metformin; Osteoblast; Osteoporosis; Diabetes mellitus

\section{Introduction}

Metformin is one of the insulin-sensitizing agents most commonly used for the management of different conditions associated with insulin-resistance, such as type 2 diabetes, metabolic syndrome and polycystic ovary syndrome. Metformin is an anti-hyperglycaemic biguanide, which has been widely used for decades. Nevertheless, the precise mechanism of action of metformin remains unclear. It is considered an insulin-sensitizing drug, lowering glycaemic levels without increasing insulin secretion (Garber, 2004). Several sites of action have been proposed for metformin, including decreased hepatic glucose output, increased peripheral glucose uptake and improved insulin secretion (Bailey and Turner, 1996). Metformin has also been shown to have several beneficial effects on cardiovascular risk factors including improvement

\footnotetext{
* Corresponding author. Cátedra de Bioquímica Patológica, Facultad de Ciencias Exactas, Universidad Nacional de La Plata. Calle 47 y 115, (1900) La Plata, Argentina.

E-mail address: cortizo@biol.unlp.edu.ar (A.M. Cortizo).
}

in lipid profile, decreased propensity towards intravascular thrombosis by lowering concentrations and activity of plasminogen activator inhibitor-1, decreasing platelet aggregation and adhesion, increasing tissue plasminogen activator activity, and improving endothelial dysfunction. In a clinical setting, metformin is an oral anti-hyperglycaemic agent which has been firmly associated with a long-term decrease in macrovascular events in type 2 diabetic patients (UKPDS 34, 1998).

Several studies have shown both in vivo and in vitro that metformin can act through activation of tyrosine kinases (Dominguez et al., 1996) and AMP-activated protein kinase (AMPK) (Zhou et al., 2001; Musi et al., 2002). AMPK phosphorylates multiple enzymes involved in several biosynthetical pathways such as acetyl-CoA carboxylase, hydroxymethylglutaryl-CoA reductase, glycogen synthase and endothelial nitric oxide synthase (eNOS). AMPK activation results in an increase in cellular glucose transport both in liver and muscle cells, and in a decrease in hepatic glucose production, cholesterol synthesis and triglyceride synthesis (Kemp et al., 1999; Chen et al., 1999; Zou et al., 2004). 
Bone is a dynamic tissue that constantly undergoes remodelling, through the precise and localized coupling of resorption (removal of aged material) with replacement by newly formed bone. This process requires the interaction between different cell types such as osteoblasts, macrophages and osteoclasts, which is regulated in turn by a variety of biochemical and mechanical factors (Hill, 1998).

Various signal-transduction mechanisms have been shown to be particularly relevant to the growth and development of bone cells. Nitric oxide (NO) is a signalling molecule constitutively produced in bone cells, and which increases in response to several stimuli such as sex hormones, mechanical strain and proinflammatory cytokines. NO is generated by the nitric oxide synthase enzymes (NOS). Two isoforms of NOS, inducible (iNOS) and endothelial (eNOS), are expressed in osteoblasts (Van't Hof and Ralston, 2001; Rubin et al., 2003). It has been previously demonstrated that eNOS knockout mice have reduced bone formation, a decrease in osteoblast number and diminished mineral apposition rate, showing that the eNOS pathway plays a central role in bone metabolism by modulating osteoblastic function (Armour et al., 2001).

Additionally, in osteoblasts, the mitogen-activated protein kinase (MAPK) pathway constitutes an important transducer of extracellular matrix-integrin signals to the nucleus (Gumbiner, 1996) and the subsequent stimulation of Cbfa1-dependent transcription (Xiao et al., 2002). Extracellular signal-regulated kinase (ERK) 1/2 (p42 and p44 MAPK) are strongly activated by growth factors, serum, bisphosphonates and estrogen in bone cells (Plotkin et al., 2005a,b). ERK1/2 are distributed throughout non-stimulated cells, but upon stimulation a significant proportion of ERK $1 / 2$ accumulates in the nucleus (Chen et al., 2005). The mechanisms involved in nuclear accumulation of ERK1/2 are still obscure, although nuclear retention, dimerization, phosphorylation and release from cytoplasmic anchors have been shown to play a role controlling cell differentiation and cell behavior (Xiao et al., 2002; Plotkin et al., 2005a,b; Roux and Blenis, 2004). In addition, it has been suggested that the kinetics of ERK phosphorylation and the length of time that P-ERKs are retained in the nucleus are responsible for pro- versus anti-apoptotic effects of estrogens on different cell types of bone (Chen et al., 2005).

In the present work, we have evaluated the effects of metformin on the growth and differentiation of osteoblasts in culture. In addition, we have also investigated possible mechanisms of action of metformin, such as the expression of eNOS and iNOS and the activation of ERK 1/2.

\section{Materials and methods}

\subsection{Materials}

Dulbecco's modified Eagle's medium (DMEM), trypsinEDTA and fetal bovine serum (FBS) were obtained from Gibco (Invitrogen, Buenos Aires, Argentina). Tissue culture disposable material was from Nunc (Tecnolab, Buenos Aires, Argentina). Goat polyclonal anti-ERK1/2 antibodies, a monoclonal anti-P-ERK antibody and polyclonal antibodies against
eNOS and iNOS were from Santa Cruz Biotechnology Inc. (Santa Cruz, CA, USA). Di-amino-bencidine, as well as Sirius red and Alizarin red S dyes were obtained from Sigma-Aldrich (Buenos Aires, Argentina). Metformin was donated by Quimica Montpellier (Buenos Aires, Argentina). All other chemicals and reagents were purchased from commercial sources and were of analytical grade.

\subsection{Cell culture and incubations}

UMR106 rat osteosarcoma cells and MC3T3E1 mouse calvaria-derived cells were grown in DMEM containing $10 \%$ FBS, $100 \mathrm{U} / \mathrm{ml}$ penicillin and $100 \mu \mathrm{g} / \mathrm{ml}$ streptomycin at $37^{\circ} \mathrm{C}$ in a 5\% $\mathrm{CO}_{2}$ atmosphere (McCarthy et al., 1997). Cells were seeded on $75-\mathrm{cm}^{2}$ flasks, subcultured using trypsin-EDTA and replated on multi-well plates. The UMR106 cell line has been shown to conserve certain characteristics of differentiated osteoblastic phenotype (Partridge et al., 1983). In the case of non-transformed MC3T3E1 cells, previous studies have demonstrated that expression of osteoblastic markers begins after culturing the cells with medium supplemented by $5 \mathrm{mM} \beta-$ glycerol-phosphate and $145 \mu \mathrm{M}$ ascorbic acid (Quarles et al., 1992). Under these culture conditions, alkaline phosphatase activity begins to be expressed after 1 week and reaches a maximum after 2 weeks, while mineralization is achieved after extending the culture to 3 weeks. However, the cells only undergo active replication during the first 5 days of incubation. For proliferation experiments with both cell lines and differentiation experiments with UMR106 osteoblasts, cells on multi-well plates were incubated in serum-free medium with different doses of metformin, during the periods of time indicated in the legends of figures. For type-I collagen secretion and mineralization experiments with MC3T3E1 osteoblasts, cells were cultured for 2 or 3 weeks, respectively, in DMEM/ FBS (with or without different doses of metformin) supplemented with $\beta$-glycerol-phosphate and ascorbic acid, changing this complete medium every 2 days. For alkaline phosphatase expression experiments with MC3T3E1 osteoblasts, cells were cultured for 2 weeks in DMEM/FBS supplemented with $\beta$ glycerol-phosphate and ascorbic acid changing the medium every 2 days, after which they were serum-starved and incubated in DMEM with or without different doses of metformin for an additional $24 \mathrm{~h}$.

\subsection{Cell proliferation assay}

Cell proliferation was determined using the crystal violet mitogenic bioassay as described previously (Okajima et al., 1992), with some modifications. Briefly, cells were grown in 48-well plates and incubated with different concentrations of metformin in serum-free DMEM, according to the figure legends. Then, the monolayers were washed with phosphatebuffered saline (PBS) and fixed with 5\% glutaraldehyde/PBS at room temperature for $10 \mathrm{~min}$. After that, they were stained with $0.5 \%$ crystal violet $/ 25 \%$ methanol for $10 \mathrm{~min}$. The dye taken up by the cells was extracted using $0.5 \mathrm{ml} /$ well $0.1 \mathrm{M}$ glycine $/ \mathrm{HCl}$ buffer, $\mathrm{pH} 3.0 / 30 \%$ methanol and transferred to 
test tubes. Absorbance was read at $540 \mathrm{~nm}$ after a convenient sample dilution. We have previously shown that, under these conditions, this colorimetric bioassay strongly correlates with cell proliferation measured by cell counting in Neubauer chambers (Cortizo and Etcheverry, 1995; Etcheverry et al., 1997).

\subsection{Assays for osteoblast differentiation and mineralization}

Osteoblastic differentiation was evaluated by two markers: alkaline phosphatase activity and the quantitation of type-I collagen production. Alkaline phosphatase activity was assayed as we have previously described (Cortizo and Etcheverry, 1995; Molinuevo et al., 2004). Briefly, the cells were incubated overnight with different doses of metformin in serum-free DMEM. The cell layer was then washed with PBS and solubilized in $0.5 \mathrm{ml} 0.1 \%$ Triton X-100. Aliquots of the total cell extract were used for protein determination by the Bradford (1976) technique. Measurement of alkaline phosphatase activity was carried out by spectrophotometric determination of initial rates of hydrolysis of para-nitrophenyl-phosphate to para-nitrophenol at $37^{\circ} \mathrm{C}$ for $10 \mathrm{~min}$. The production of para-nitrophenol was determined by absorbance at $405 \mathrm{~nm}$. Under these experimental conditions, the product formation was linear for $15 \mathrm{~min}$. Collagen production was evaluated with a Sirius red-based colorimetric micro-assay (Tullberg-Reinert and Jundt, 1999): cells were fixed in Bouin's fluid for $1 \mathrm{~h}$, washed with water and stained with Sirius red dye for $1 \mathrm{~h}$. The stained material was dissolved in $0.1 \mathrm{~N}$ sodium hydroxide and absorbance read at $550 \mathrm{~nm}$. Mineralized nodules in the extracellular matrix of MC3T3E1 osteoblasts were stained with Alizarin red S (Ueno et al., 2001). The dye taken up by extracellular calcium deposits was dissolved in $0.1 \mathrm{~N}$ sodium hydroxide and quantified spectrophotometrically at $548 \mathrm{~nm}$.

\subsection{Western blot analysis of P-ERK and NOS}

Metformin-induced regulation of ERK 1/2 activation, as well as eNOS and iNOS expression, were evaluated by Western blot (Barrio et al., 2003; Cortizo et al., 2000). In these experiments, osteoblastic cells growing in serum-free medium with the addition of different concentrations of metformin were lysated in Laemmli's buffer (Laemmli et al., 1979) and the protein content was evaluated by the method of Lowry (Lowry et al., 1951). These lysates were heated at $100^{\circ} \mathrm{C}$ for $3 \mathrm{~min}$ and aliquots corresponding to $30 \mu \mathrm{g}$ of total protein were subjected to $12 \%$ SDS-PAGE. The separated proteins were then electrotransferred to PVDF membranes. After washing and blocking, the membranes were incubated with a monoclonal anti-P-ERK antibody, a polyclonal antibody that recognizes both phosphorylated and unphosphorylated ERK1/2, or polyclonal antibodies against eNOS or iNOS. Blots were developed using di-aminobencidine-peroxidase staining. The intensity of the specific bands was quantified by densitometry after scanning of the membranes. Images were analysed using the Scion-beta 2 program.

\subsection{Localization of P-ERK and i/eNOS by immunofluorescence analysis}

Cells grown on coverslips with or without different concentrations of metformin were fixed in $4 \%$ para-formaldehyde in PBS, blocked and incubated with a monoclonal anti-PERK antibody, polyclonal anti-iNOS or polyclonal anti-eNOS (1:200 each) at $4{ }^{\circ} \mathrm{C}$ for $16 \mathrm{~h}$. After washing, a secondary FITClabelled antibody (1:200) was added for $2 \mathrm{~h}$ at room temperature. Coverslips were mounted in a Vectastain mounting liquid and the distribution of the specific protein was imaged using a Nikon-5000 fluorescence microscope and a digital camera.

\subsection{Statistical analysis}

Three independent experiments were run for each experimental condition. Results are expressed as the mean \pm S.E.M. One-way ANOVA with Dunnett's post-test was performed using GraphPad InStat version 3.05.GraphPad Software, San Diego, California, USA. $P<0.05$ was accepted as statistically significant when compared with untreated control $(0 \mu \mathrm{M}$ metformin).

\section{Results}

\subsection{Metformin regulates osteoblastic growth, differentiation and mineralization}

To investigate the effect of metformin on growth and differentiation of osteoblasts, two osteoblast-like cell lines (MC3T3E1 and UMR106) were used. Treatment of each cell line with metformin $(25-500 \mu \mathrm{M})$ for $24 \mathrm{~h}$ led to a dosedependent increase of cell proliferation as determined by the crystal violet bioassay. Fig. 1A shows that maximal stimulation was reached at a concentration of $200 \mu \mathrm{M}(115 \%$ of basal) for both cell lines.

To examine the effect of metformin on osteoblast differentiation, alkaline phosphatase activity and type-I collagen production were evaluated. Metformin (25$500 \mu \mathrm{M})$ had no effect on alkaline phosphatase activity in UMR106 cells (data not shown). Pre-osteoblastic MC3T3E1 cells were differentiated in the presence of ascorbic acid and $\beta$-glycerol-phosphate during 2 weeks. After this culture period, differentiated MC3T3E1 osteoblasts were treated with metformin $(25-500 \mu \mathrm{M})$ for $24 \mathrm{~h}$. Fig. 1B shows that the anti-diabetic drug significantly stimulated alkaline phosphatase in a dose-dependent manner, with a maximum at $100 \mu \mathrm{M}$ concentration ( $145 \%$ of basal). In addition, metformin dose-dependently stimulated the production of a type-I collagenous matrix both by the UMR106 (113\% of basal) and the MC3T3E1 cell lines (128\% of basal) (Fig. 1C). Furthermore, the effect of this anti-diabetic compound on the ability of MC3T3E1 cells to mineralize the matrix was assessed after 3 weeks of culture in the presence of $\beta$-glycerol phosphate and ascorbic acid. Fig. 1D shows that metformin at $500 \mu \mathrm{M}$ significantly stimulated $(385 \%$ basal $)$ calcium 
(A)

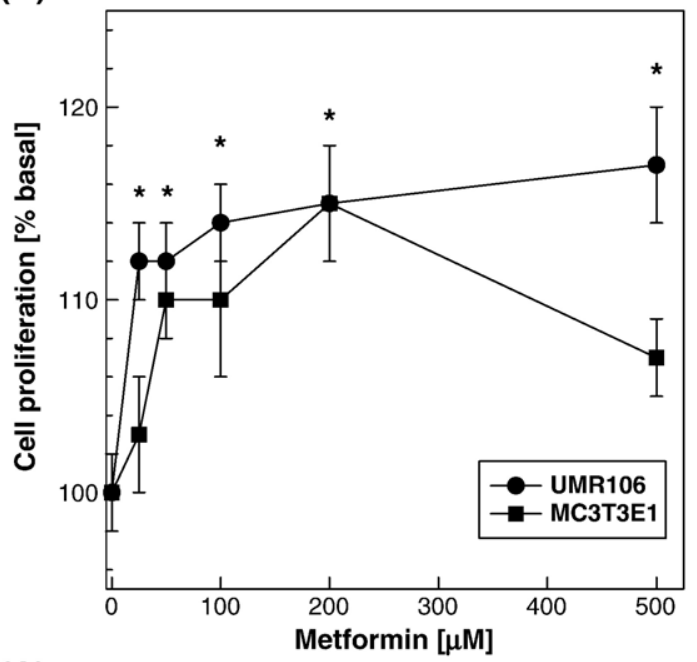

(C)

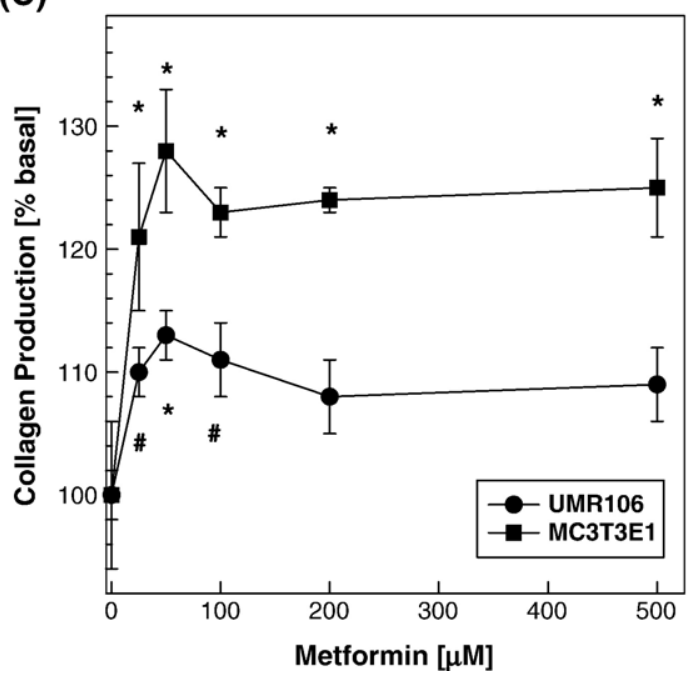

(B)

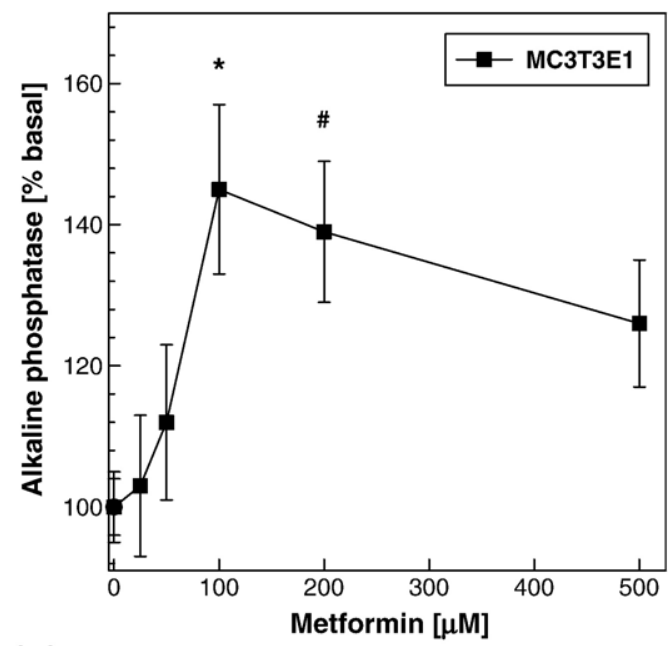

(D)

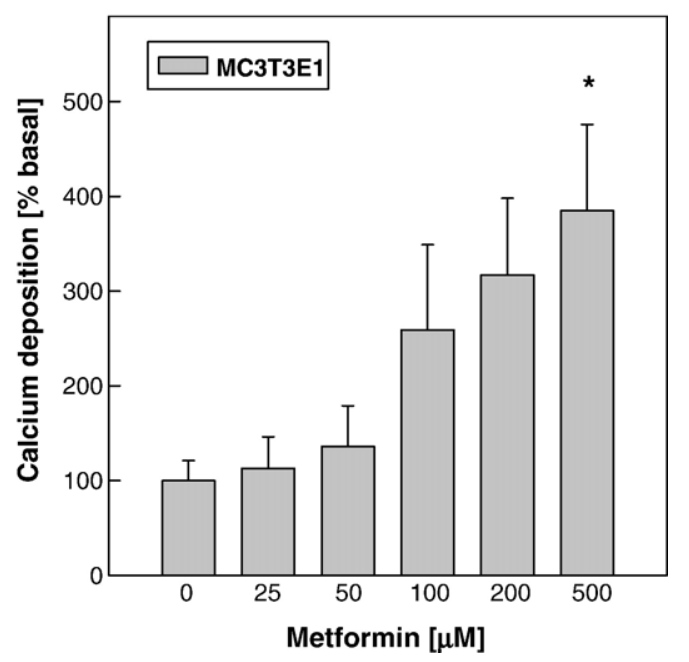

Fig. 1. Effect of metformin on proliferation, differentiation and mineralization of osteoblastic cells. (A) MC3T3e1 and UMR106 osteoblasts were incubated in serumfree media in the presence of different concentrations of metformin for $24 \mathrm{~h}$. Subsequently, proliferation was evaluated by the crystal violet bioassay. (B) MC3T3E1 pre-osteoblasts were differentiated during 2 weeks in the presence of $\beta$ GP and AA. ALP was assessed after 24-h incubation in serum-free media with different doses of metformin, as described in Section 2. (C) MC3T3E1 cells were differentiated for 2 weeks in $\beta$ GP and AA-supplemented media and different concentrations of metformin, and UMR106 were incubated for $48 \mathrm{~h}$ in serum-free media with different doses of metformin. After these culture periods, type-I collagen production was evaluated by Sirius red staining. (D) MC3T3E1 osteoblasts were cultured for 3 weeks in $\beta$ GP and AA-supplemented media and different concentrations of metformin. Mineralized nodules were quantitated by Alizarin-Red S staining, as described in Section 2. Data represent mean \pm S.E.M. $\# p<0.05$, $* p<0.01$, when compared with untreated control.

deposition in mineralized nodules as assessed by the Alizarin red S staining.

Our results indicate that metformin can act as a weak mitogen in osteoblastic cells, and that the long-term exposure of osteoblastic cultures to metformin induces osteogenic effects.

\subsection{Metformin acutely induces ERK phosphorylation and its subcellular redistribution}

P42/44 MAPK is an important signal transduction pathway involved in several growth factor effects. In order to investigate the mechanism by which metformin affects osteoblastic growth and differentiation, osteoblastic cells were treated with $1 \mathrm{mM}$ metformin for different periods of time. At the end of this incubation, the activation/phosphorylation of ERK was analyzed by Western immunoblot and the intracellular localization of P-ERK by immunofluorescence, using a specific anti-P-ERK antibody. In MC3T3E1 osteoblasts, metformin significantly increased P-ERK after $5 \mathrm{~min}$, an activation that was sustained for $60 \mathrm{~min}$ and decreased to basal levels after 90 min of incubation (Fig. 2A). However, there was no change in total ERK protein, as assessed by immunoblots using an anti-ERK antibody. Immunofluorescence experiments showed that P-ERK was mainly present in the cytoplasm with a punctate-granular staining in the control non-treated osteoblasts (Fig. 2B), although a minor proportion of fluorescence appeared associated with the nucleus ( $22 \%$ of stained cells). After 
(A)
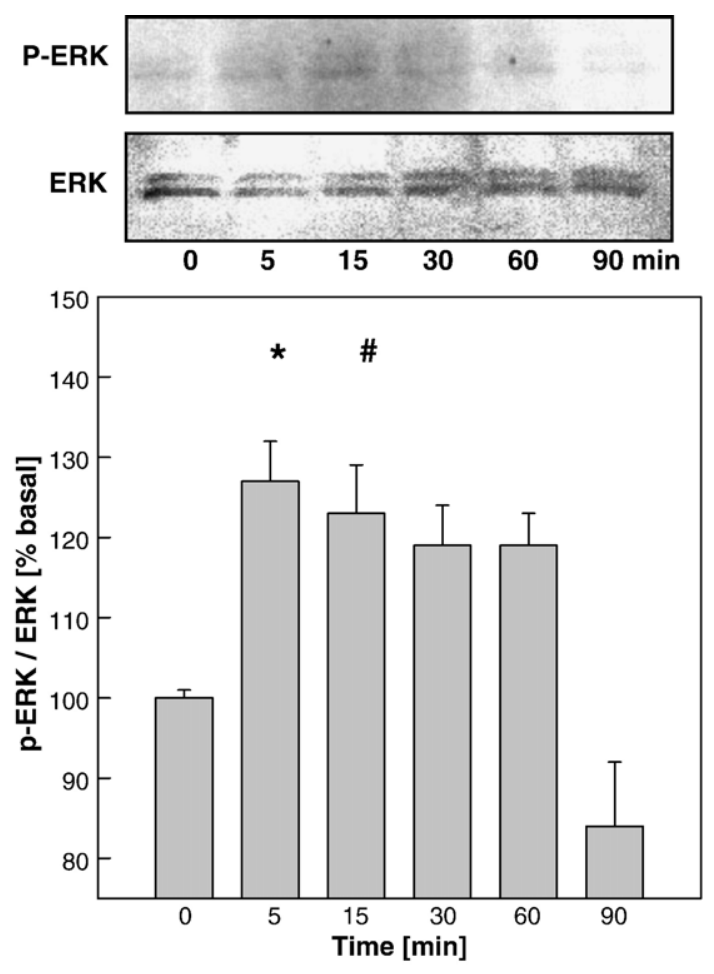

(B)
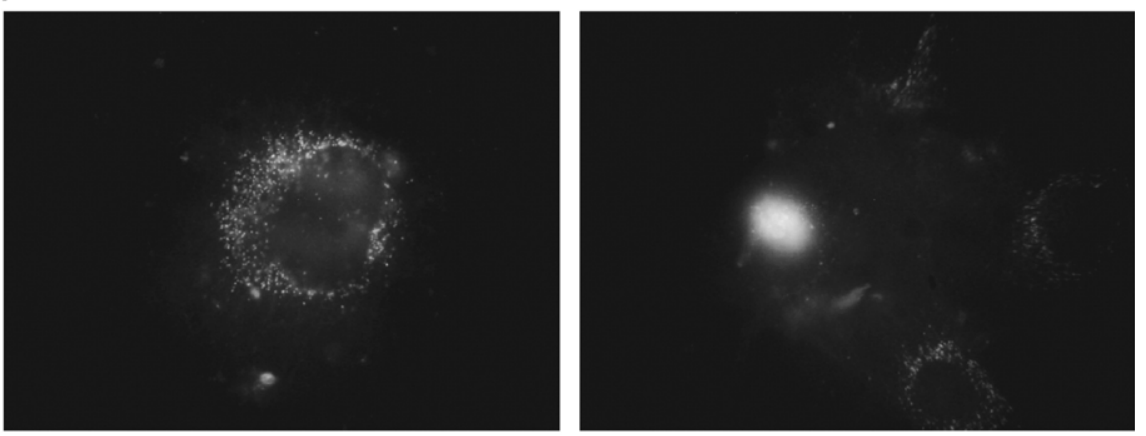

$0 \mathrm{~min}$

$15 \mathrm{~min}$
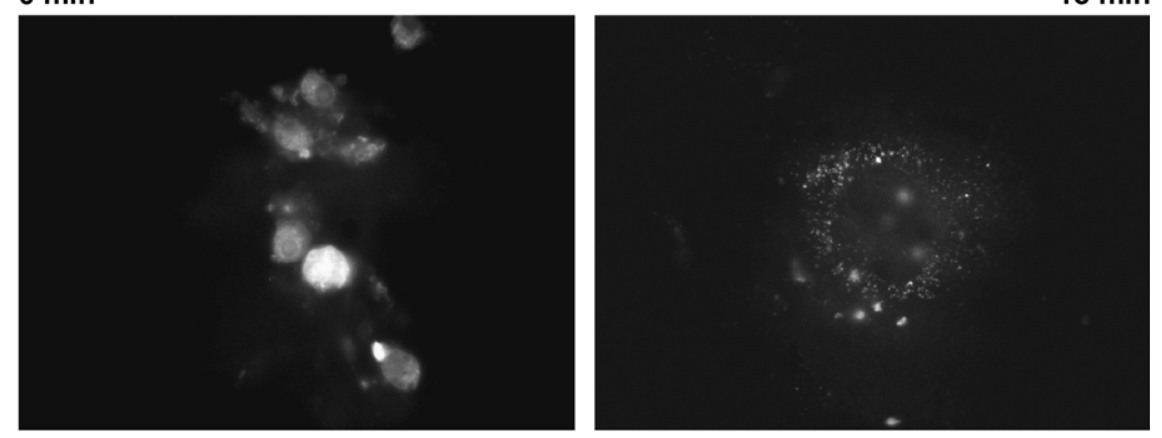

$30 \min$

$90 \mathrm{~min}$

Fig. 2. Effect of metformin on ERK activation and distribution. MC3T3E1 cells were serum starved for $24 \mathrm{~h}$ and incubated with $1 \mathrm{mM}$ metformin for different periods of time. (A) Cell lysates were collected, and both total and phosphorylated ERK 1/2 phosphorylation was assessed by Western immunoblot analysis. (B) P-ERK localization in the cells was assessed by indirect immunofluorescence. At the indicated times, cells were fixed and incubated with an anti-P-ERK antibody. Obj. 100×. Data represent mean \pm S.E.M. $\# p<0.05, * p<0.01$, when compared with untreated control.

15-30 min incubation with $1 \mathrm{mM}$ metformin, an increase in the nuclei-associated fluorescence was observed $(43 \%$ of stained cells). This effect disappeared after 90-min incubation. In the case of UMR106 osteosarcoma cells, treatment with metformin induced effects on ERK phosphorylation and redistribution, which were identical to those observed for MC3T3E1 osteoblasts (data not shown). All in all, these results suggest that metformin induces the activation and redistribution of P-ERK in a transient manner in osteoblastic cells. 


\subsection{NOS expression is regulated by metformin}

Nitric oxide is known to be implicated in the metabolism of bone, especially as a mediator of cytokine effects on the remodeling of bone tissue. Thus, we investigated whether metformin had any effect on the expression of eNOS and iNOS in MC3T3E1 osteoblasts, as evaluated by Western immunoblot and immunofluorescence.

In the non-transformed MC3T3E1 osteoblasts, 24-h incubation with $50-500 \mu \mathrm{M}$ metformin stimulated the expression of both NOS isoforms in a dose-dependent manner (Fig. 3A and B). By immunofluorescent analysis of control MC3T3E1 cells, eNOS was shown to localize principally in the cytoplasm in a diffuse and patchy pattern, while iNOS was also cytoplasmic but with a more punctuate pattern. Incubation with $100 \mu \mathrm{M}$ metformin increased both eNOSand iNOS-associated fluorescence, showing a more homogeneous cytoplasmic distribution (Fig. 3A). A dose-dependent effect of metformin on eNOS and iNOS expression in MC3T3E1 cells was shown by western immunoblot (Fig 3B). Endothelial NOS stimulation by metformin was biphasic, showing a peak at $100 \mu \mathrm{M}$ concentration. On the other hand,
(A)
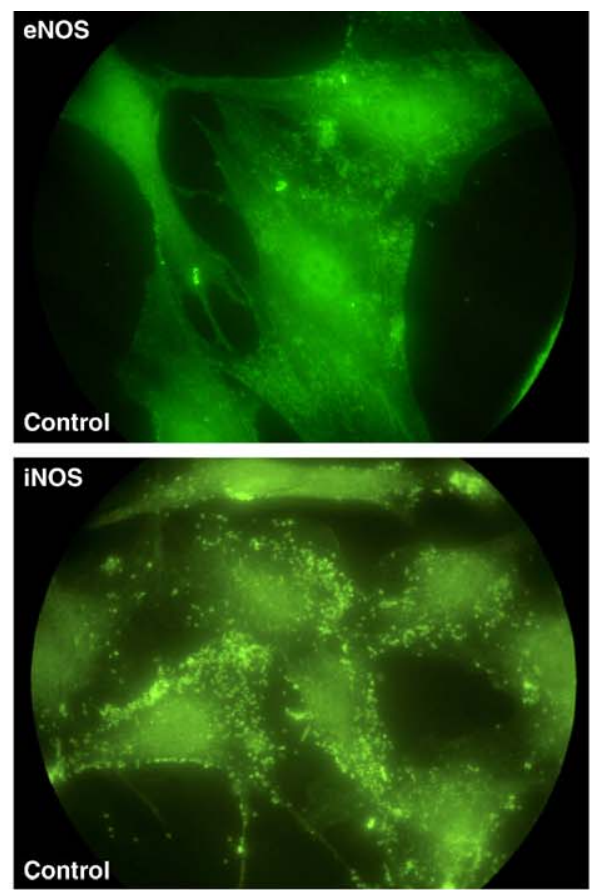

(B)
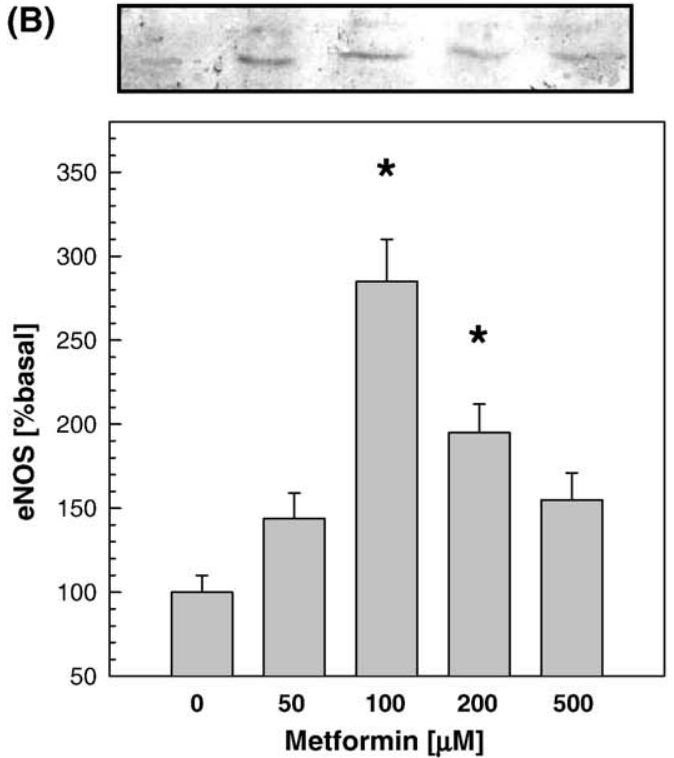
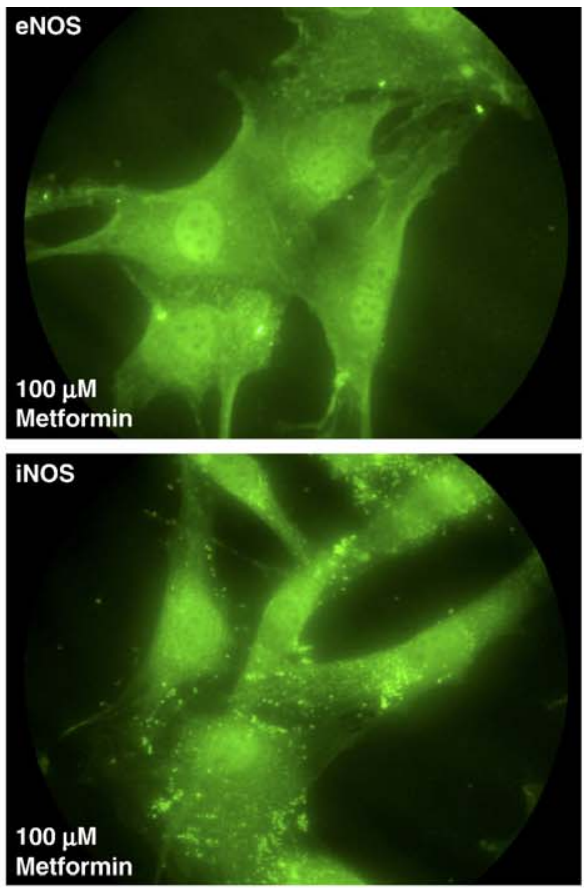

\section{Metformin}

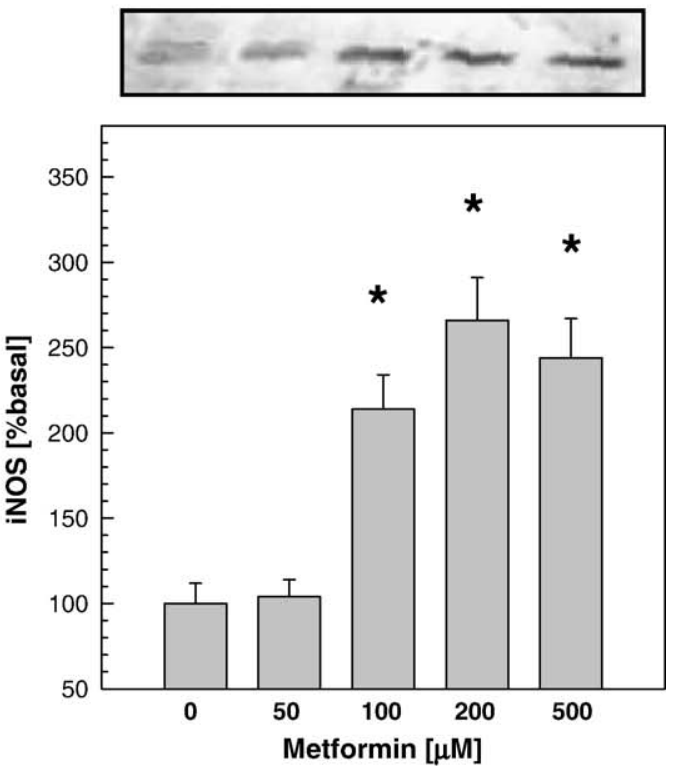

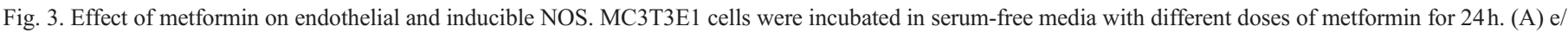

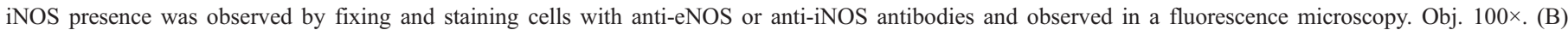

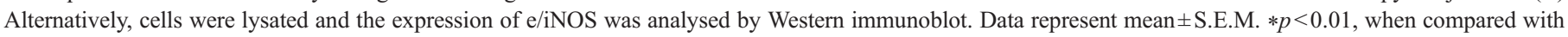
untreated control. 
the effect of metformin on iNOS expression was dosedependent, reaching a plateau after $200 \mu \mathrm{M}$ doses.

\section{Discussion}

Osteoporosis, one of the most prevalent metabolic bone diseases in developed countries, is a major public health problem due to its association with fragility fractures (Chau et al., 2003). Several studies have reported skeletal involvement in diabetic patients; however, the mechanisms underlying this association are poorly understood (Chau et al., 2003; Carnevale et al., 2004). Krakauer et al. (1995) showed in a 12-year follow-up study that osteoblastic extracellular matrix formation and mineralization were reduced by $70-75 \%$ in patients with diabetes mellitus. These investigators proposed that this impaired osteoblastic function could be the cause of the osteopenia observed in their series of type 1 diabetic patients and of the decrease in bone remodeling observed in their series of type 2 diabetic patients. Previous research has identified as a possible cause for these diabetes-associated bone alterations, the pathological accumulation of advanced glycation end-products on bone extracellular matrix proteins (McCarthy et al., 1997; Tomasek et al., 1994; Katayama et al., 1996). Advanced glycation end-products are irreversibly and non-enzymatically formed on long-lived proteins, accumulate in diabetic patients as a consequence of chronic hyperglycemia and oxidative stress, and are thought to be partly responsible for the macrovascular and microvascular complications of diabetes mellitus (Vlassara and Palace, 2002). In previous studies, we have shown that advanced glycation end-productsmodified type-I collagen decreases the differentiation, mineralization and long-term survival of MC3T3E1 osteoblasts in culture, probably through the interaction of advanced glycation end-products with specific receptors (McCarthy et al., 2001a,b). Advanced glycation end-products have also been shown to prevent the differentiation of human mesenchymal stem cells into bone-forming osteoblasts (Kume et al., 2005).

In view of the association which has been established between long-standing diabetes mellitus and the loss of bone mineral density and/or quality, it is clinically relevant to investigate the effects of widely used anti-diabetic drugs on the metabolism of bone cells. Recently, detrimental effects of the insulin-sensitizing drug rosiglitazone have been demonstrated by in vitro and in vivo studies on bone tissue: rosiglitazone was shown to promote commitment of osteoprogenitor cells to the adipocyte lineage while inhibiting osteoblastogenesis and bone formation (Lecka-Czernik et al., 1999, 2002).

There have been no previous studies investigating the possible actions on bone cells of metformin, an insulinsensitizing drug widely used for the treatment of diabetic patients. In the present study, we have studied the effects of metformin on the growth and development of two osteoblastlike cell lines. Our present results demonstrate for the first time that metformin exerts osteogenic actions on osteoblasts in culture. Evidence for this notion is based on several observations: (a) metformin acts as a weak mitogen, stimulating osteoblastic proliferation; (b) although metformin does not affect alkaline phosphatase activity in the UMR106 osteosarcoma cell line, it increases this marker of osteoblastic differentiation in the non-transformed MC3T3E1 cells; (c) metformin dose-dependently stimulates type-I collagen production in both cell lines; and (d) after 3 weeks of culture, metformin strongly induces the formation of nodules of mineralization in MC3T3E1 osteoblasts. Thus, in our in vitro experimental conditions, this anti-diabetic drug not only induces osteoblast growth and differentiation, but also has the ability to increase mineralization of the extracellular matrix. In vivo studies are needed to investigate whether metformin also has bone-preserving effects opposing the development of diabetic osteopenia and osteoporosis.

Different authors have investigated the effects of metformin on the proliferation of other cell types. This biguanide did not influence cell proliferation in cultured arterial smooth muscle cells, human dermal fibroblasts, umbilical artery-derived smooth muscle cells or 3T3 fibroblastic cells (Peuler et al., 1996; Petty and Pearson, 1992). In the present in vitro study, we present data that clearly shows a proliferative effect of metformin on osteoblast-like cells, in a concentration range similar to that used by other authors (Peuler et al., 1996; Petty and Pearson, 1992).

Our present results also demonstrate that metformin induces osteoblastic differentiation, as assessed by two well-established markers: alkaline phosphatase and type-I collagen production. However, no effect was observed by metformin on the alkaline phosphatase activity of UMR 106 cells. This could be due to the fact that UMR106 osteosarcoma cells greatly over-express alkaline phosphatase in their basal state (100-fold greater than MC3T3E1 cells), so it is not surprising that metformin has no effect on alkaline phosphatase activity in these cells. In addition, a sharp increase was observed in the formation of nodules of mineralization in long-term cultures of MC3T3E1 osteoblasts treated with metformin. In our experimental model, these osteogenic effects of metformin may be mediated by the secretion of soluble cytokines and/or growth factors into the conditioned media. Further studies are necessary to investigate if metformin addition is capable of modifying growth factor secretion by osteoblasts in culture.

In order to investigate possible intracellular mechanisms which could be mediating the osteogenic effects exerted by metformin on osteoblasts, we first evaluated the ERK pathway. Phosphorylation (activation) and intracellular redistribution of ERK-1/2 have been identified as established transduction mechanisms for various agents acting on bone cells, such as bisphosphonates, estrogen, parathyroid hormone and mechanical stimulation (Plotkin et al., 2005a,b, 2002). The data presented in this study shows that ERK-1/2 is phosphorylated after metformin treatment, in a time-dependent manner, in both cell lines. In addition, a nuclear translocation of P-ERK was observed after incubating the osteoblasts with metformin for 15-30 min, with P-ERK returning to the cytoplasm after 90 min. Duration and localization of MAPK signals are normally regulated during the proliferation and differentiation of many cell types (Pouyssegur et al., 2002). In particular, they 
have been implicated in the anti- and pro-apoptotic effects of estrogen on different bone cell types (Chen et al., 2005). To address this issue in particular, in the present study, we also investigated the possible effect of metformin on the apoptosis of osteoblasts in culture. However, we were unable to find any effect of metformin per se on the apoptosis of UMR106 and MC3T3E1 osteoblastic cell lines (data not shown). Thus, the metformin-induced ERK-1/2 phosphorylation and relocalization could be associated with the observed increase in osteoblastic growth and development. The MAPK signaling pathway is known to regulate the activity of different transcription factors, which are subsequently involved in the regulation of proliferation, apoptosis and differentiation (Burdon et al., 2002). Further studies are necessary to evaluate this possibility.

Another signaling pathway which could be affected by metformin in osteoblasts is the enzymatic generation of NO by endothelial and inducible isoforms of NOS. Nitric oxide is constitutively produced by osteoblasts, and has been shown to modulate bone remodeling (Van't Hof and Ralston, 2001; Collin-Osdoby et al., 1995). Our group and other investigators have previously suggested that NO has biphasic effects on osteoblasts (Cortizo et al., 2000; Collin-Osdoby et al., 1995; Mancini et al., 2000). Constitutive or low levels of NO can be effective mediators of osteoblastic growth and differentiation; however, overproduction of NO leads to osteoblastic injury. We have also shown that MC3T3E1 osteoblasts express readily detectable levels of eNOS and iNOS (Cortizo et al., 2000). In the present study, we demonstrate that metformin increases the expression of both NOS isoforms in a dose-dependent manner, with maximal stimulation at $100 \mu \mathrm{M}$ of metformin. This effect was found to closely parallel the metformin-induced increase in osteoblastic proliferation, suggesting a link between NO production and cell growth in this cell line. Interestingly, other authors have shown that hepatocyte growth factor can exert effects on human endothelial cells, by sequentially inducing eNOS expression, NO production and MAPK phosphorylation (activation), which in turn increases cellular proliferation (Kuhlmann et al., 2005).

In conclusion, these results show for the first time a direct osteogenic effect of metformin on osteoblasts in culture and suggest that these actions could be partly mediated by activation/redistribution of ERK-1/2 and induction of e/iNOS. These potentially bone-preserving effects of metformin could be particularly relevant in the clinical setting of diabetic osteopenia and osteoporosis.

\section{Acknowledgments}

This study was partially supported by grants from Universidad Nacional de La Plata, Comisión de Investigaciones Científicas de la Provincia de Buenos Aires (CICPBA) and Novo Nordisk Laboratories, Argentina. We wish to thank Química Montpellier Argentina for their generous donation of metformin and Dr. Claudio Gonzalez for his kind collaboration with the statistical analysis of the data. AMC is a member of the Carrera del Investigador, CICPBA.

\section{References}

Armour, K.E., Armour, K.J., Gallagher, M.E., Gödecke, A., Helfrisch, M.H., Reid, D.M., Ralston, S.H., 2001. Defective bone formation and anabolic response to exogenous estrogen in mice with targeted disruption of endothelial nitric oxide synthase. Endocrinology 142, 760-766.

Bailey, C.J., Turner, R.C., 1996. Metformin. N. Engl. J. Med. 334, 574-579.

Barrio, D.A., Williams, P.A., Cortizo, A.M., Etcheverry, S.B., 2003. Synthesis of a new vanadyl (IV) complex with trehalose (TreVO): insulin-mimetic activities in osteoblast-like cells in culture. J. Biol. Inorg. Chem. 8, 459-468.

Bradford, M.M., 1976. A rapid and sensitive method for the quantitation of microgram quantities of protein utilizing the principle of protein-dye binding. Anal. Biochem. 72, 248-254.

Burdon, T., Smith, A., Savatier, P., 2002. Signaling, cell cycle and pluripotency in embryonic stem cells. Trends Cell Biol. 12, 432-438.

Carnevale, V., Romagnoli, E., D'Erasmo, E., 2004. Skeletal involvement in patients with diabetes mellitus. Diabetes Metab. Res. Rev. 20, 196-204.

Chau, D.L., Edelman, S.V., Chandran, M., 2003. Osteoporosis and diabetes. Curr. Diabetes Rep. 3, 37-42.

Chen, Z.P., Mitchelhill, K.I., Michell, B.J., Stapleton, D., Rodriguez-Crespo, I., Witters, L.A., Power, D.A., Ortiz de Montellano, P.R., Kemp, B.E., 1999. AMP-activated protein kinase phosphorylation of endothelial NO synthase. FEBS Lett. 443, 285-289.

Chen, J.R., Plotkin, L.I., Aguirre, J.I., Han, L., Jilka, R.L., Kousteni, S., Bellido, T., Manolagas, S.C., 2005. Transient versus sustained phosphorylation and nuclear accumulation of ERKs underlie anti- versus pro-apoptotic effects of estrogens. J. Biol. Chem. 280, 4632-4638.

Collin-Osdoby, P., Nickols, G.A., Osdoby, P., 1995. Bone cell function, regulation, and communication: a role for nitric oxide. J. Cell. Biochem. 57, 399-408.

Cortizo, A.M., Etcheverry, S.B., 1995. Vanadium derivatives act as growth factor-mimetic compounds upon differentiation and proliferation of osteoblast-like UMR106 cells. Mol. Cell. Biochem. 145, 97-102.

Cortizo, A.M., Caporossi, M., Lettieri, G., Etcheverry, S.B., 2000. Vanadateinduced nitric oxide production: role in osteoblast growth and differentiation. Eur. J. Pharmacol. 400, 279-285.

Dominguez, L.J., Davidoff, A.J., Srinivas, P., Standley, P., Walsh, M.F., Sowers, J.R., 1996. Effects of metformin on tyrosine kinase activity, glucose transport and intracellular calcium in rat vascular smooth muscle. Endocrinology 137, 113-121.

Etcheverry, S.B., Crans, D.C., Keramidas, A.D., Cortizo, A.M., 1997. Insulinmimetic action of vanadium compounds on osteoblast-like cells in culture. Arch. Biochem. Biophys. 338, 7-14.

Garber, A., 2004. Metformin and other biguanides: pharmacology and therapeutic usage, In: DeFronzo, R.A., Ferrannini, E., Keen, H., Zimmet, P. (Eds.), International In: International Textbook of Diabetes Mellitus, third edition. John Wiley \& Sons, Ltd., pp. 851-865.

Gumbiner, B.M., 1996. Cell adhesion: the molecular basis of tissue architecture and morphogenesis. Cell 84, 345-357.

Hill, P.A., 1998. Bone remodelling. Br. J. Orthod. 25, 101-107.

Katayama, Y., Akatsu, T., Yamamoto, M., Kugai, N., Nagata, N., 1996. Role of non-enzymatic glycosylation of type I collagen in diabetic osteopenia. J. Bone Miner. Res. 11, 931-937.

Kemp, B.E., Mitchelhill, K.I., Stapleton, D., Michell, B.J., Chen, Z.P., Witters, L.A., 1999. Dealing with energy demand: the AMP-activated protein kinase. Trends Biochem. Sci. 24, 22-25.

Krakauer, J.C., McKenna, M.J., Buderer, N.F., Rao, D.S., Whitehouse, F.W., Parfitt, A.M., 1995. Bone loss and bone turnover in diabetes. Diabetes 44, 775-782.

Kuhlmann, C.R., Schaefer, C.A., Fehsecke, A., Most, A.K., Tillmanns, A., Erdogan, A., 2005. A new signaling mechanism of hepatocyte growth factor-induced endothelial proliferation. J. Thromb. Haemost. 3, 2089-2095

Kume, S., Kato, S., Yamagishi, S.-I., Inagaki, Y., Ueda, S., Arima, N., Okawa, T., Kojiro, M., Nagata, K., 2005. Advanced glycation end-products attenuate human mesenchymal stem cells and prevent cognate differentiation into adipose tissue, cartilage and bone. J. Bone Miner. Res. 20, 1647-1658. 
Laemmli, U.K., Beguin, F., Gujer-Kellenberger, G., 1979. A factor preventing the major head protein of bacteriophage T4 from random aggregation. J. Mol. Biol. 47, 69-85.

Lecka-Czernik, B., Gubrij, I., Moerman, E.J., Kajkenova, O., Lipschitz, D.A., Manolagas, S.C., Jilka, R.L., 1999. Inhibition of Osf2/Cbfa1 expression and terminal osteoblast differentiation by PPARgamma2. J. Cell. Biochem. 74, 357-371.

Lecka-Czernik, B., Moerman, E.J., Grant, D.F., Lehmann, J.M., Manolagas, S. C., Jilka, R.L., 2002. Divergent effects of selective peroxisome proliferatorsactivated receptor- $\gamma 2$ ligands on adipocyte versus osteoblast differentiation. Endocrinology 143, 2376-2384.

Lowry, O.H., Rosebrough, N.J., Farr, A.L., Randall, R.J., 1951. Protein measurement with the Folin phenol reagent. J. Biol. Chem. 193, 265-275.

Mancini, L., Moradi-Bidhendi, N., Becherini, L., Martineti, V., MacIntyre, I.N., 2000. The biphasic effects of nitric oxide in primary rat osteoblasts are cGMP dependent. Biochem. Biophys. Res. Commun. 274, 477-481.

McCarthy, A.D., Etcheverry, S.B., Bruzzone, L., Cortizo, A.M., 1997. Effects of advanced glycation end-products on the proliferation and differentiation of osteoblast-like cells. Mol. Cell. Biochem. 170, 43-51.

McCarthy, A.D., Etcheverry, S.B., Bruzzone, L., Lettieri, G., Barrio, D.A., Cortizo, A.M., 2001a. Non-enzymatic glycosylation of a type-I collagen matrix: effects on osteoblastic development and oxidative stress. BMC Cell Biol. 2, 16.

McCarthy, A.D., Etcheverry, S.B., Cortizo, A.M., 2001b. Advanced glycation endproduct-specific receptors in rat and mouse osteoblast-like cells: regulation with stages of differentiation. Acta Diabetol. 36, 45-52.

Molinuevo, M.S., Barrio, D.A., Cortizo, A.M., Etcheverry, S.B., 2004. Antitumoral properties of two new vanadyl (IV) complexes on osteoblasts in culture. Role of apoptosis and oxidative stress. Cancer Chemother. Pharmacol 53, 163-172.

Musi, N., Hirshman, M.F., Nygren, J., Svanfeldt, M., Bavenholm, P., Rooyackers, O., Zhou, G., Williamson, J.M., Ljunqvist, O., Efendfic, S., Moller, D.E., Thorell, A., Goodyear, L.J., 2002. Metformin increases AMPactivated protein kinase activity in skeletal muscle of subjects with type 2 diabetes. Diabetes 51, 2074-2081.

Okajima, T., Nakamura, K., Zhang, H., Ling, N., Tanabe, T., Yasuda, T., Rosenfeld, R.G., 1992. Sensitive colorimetric bioassays for insulin-like growth factor (IGF) stimulation of cell proliferation and glucose consumption: use in studies of IGF analogs. Endocrinology 130, 2201-2212.

Partridge, N.C., Alcorn, D., Michelangeli, V.P., Ryan, G., Martin, T.J., 1983. Morphological and biochemical characterization of four clonal osteogenic sarcoma cell lines of rat origin. Cancer Res. 43, 4308-4314.

Petty, R.G., Pearson, J.D., 1992. The influence of hypoglycemic agents on the growth and metabolism of human endothelial cells. Diabet. Med. 9, 30-37.

Peuler, J.D., Phare, S.M., Iannucci, A.R., Hodorek, M.J., 1996. Differential inhibitory effects of antidiabetic drugs on arterial smooth muscle cell proliferation. Am. J. Hypertens. 9, 188-192.

Plotkin, L.I., Manolagas, S.C., Bellido, T., 2002. Transduction of cell survival signals by connexin-43 hemichannels. J. Biol. Chem. 277, 8648-8657.

Plotkin, L.I., Aguirre, J.I., Kousteni, S., Manolagas, S.C., Bellido, T., 2005a. Bisphosphonates and estrogens inhibit osteocyte apoptosis via distinct molecular mechanisms downstream of extracellular signal-regulated kinase activation. J. Biol. Chem. 280, 7317-7325.

Plotkin, L.I., Mathov, I., Aguirre, J.I., Parfitt, A.M., Manolagas, S.C., Bellido, T., 2005b. Mechanical stimulation prevents osteocyte apoptosis: requirement of integrins, Src kinases, and ERKs. Am. J. Physiol.: Cell Physiol. 289, C633-C643.

Pouyssegur, J., Volmat, V., Lenormand, P., 2002. Fidelity and spatio-temporal control in MAP kinase (ERKs) signalling. Biochem. Pharmacol. 64, 755-763.

Quarles, L.D., Yohay, D.A., Lever, L.W., Caton, R., Wenstrup, R.J., 1992. Distinct proliferative and differentiated stages of murine MC3T3-E1 cells in culture: an in vitro model of osteoblast development. J. Bone Miner. Res. 7 , 683-692.

Roux, P.P., Blenis, J., 2004. ERK and p38 MAPK-activated protein kinases: a family of protein kinases with diverse biological functions. Microbiol. Mol. Biol. Rev. 68, 320-344.

Rubin, J., Murphy, T., Zhu, L., Roy, E., Nanes, M.S., Fan, X., 2003. Mechanical strain differentially regulates endothelial nitric oxide synthase and receptor activator of nuclear $\kappa \mathrm{B}$ ligand expression via ERK 1/2 MAPK. J. Biol. Chem. 278, 34018-34025.

Tomasek, J.J., Meyers, S.W., Basinger, J.B., Green, D.J., Shew, R.L., 1994. Diabetic and age-related enhancement of collagen-linked fluorescence in cortical bones of rats. Life Sci. 55, 855-861.

Tullberg-Reinert, H., Jundt, G., 1999. In situ measurement of collagen synthesis by human bone cells with a Sirius red-based colorimetric microassay: effects of transforming growth factor beta2 and ascorbic acid 2-phosphate. Histochem. Cell Biol. 112, 271-276.

Ueno, A., Kitase, Y., Moriyama, K., Inoue, H., 2001. MC3T3E1-conditioned medium-induced mineralization by clonal rat dental pulp cells. Matrix Biol. 20, 347-355.

UKPDS 34, 1998. Effect of intensive blood-glucose control with metformin on complications in overweight patients with type 2 diabetes (UKPDS 34). UK Prospective Diabetes Study (UKPDS) Group. Lancet 352, 854-865.

Van't Hof, R.J., Ralston, S., 2001. Nitric oxide and bone. Immunology 103, 255-261.

Vlassara, H., Palace, M.R., 2002. Diabetes and advanced glycation endproducts. J. Intern. Med. 251, 87-101.

Xiao, G., Gopalakrishnan, R., Jiang, D., Reith, E., Benson, M.D., Franceschi, R. T., 2002. Bone morphogenetic proteins, extracellular matrix, and mitogenactivated protein kinase signalling pathways are required for osteoblastspecific gene expression and differentiation in MC3T3-E1 cells. J. Bone Miner. Res. 17, 101-110.

Zhou, G., Myers, R., Li, Y., Chen, Y., Shen, X., Fenyk-Melody, J., Wu, M., Ventre, J., Doebber, T., Fujii, N., Musi, N., Hirshman, M.F., Goodyear, L.J., Moller, D.E., 2001. Role of AMP-activated protein kinase in mechanism of metformin action. J. Clin. Invest. 108, 1167-1174.

Zou, M., Kirkpatrick, S.S., Davis, B.J., Nelson, J.S., Wiles, W.G., Schlattner, U., Neumann, D., Brownlee, M., Freeman, M., Goldman, M.H., 2004. Activation of the AMP-activated protein kinase by the anti-diabetic drug metformin in vivo. J. Biol. Chem. 279, 43940-43951. 